\title{
OPTIMIZACIÓN DE PROGRAMAS MATEMÁTICOS CON PROGRAMACIÓN DINÁMICA
}

\author{
OPTIMIZATION OF MATHEMATICAL PROGRAMS WITH DYNAMIC \\ PROGRAMMING
}

\author{
'Jhony Alfonso Chávez Delgado, ${ }^{2}$ Luis César Méndez Avalos, ${ }^{3}$ Eduardo Rodríguez Delgado, \\ ${ }^{4}$ Luis Asunción López Puycán
}

\section{RESUMEN}

La programación dinámica deterministica es un método de optimización muy útil para descomponer programas matemáticos grandes y complejos en etapas, en la que cada cual incluye un subproblema de una sola variable que es individualmente más fácil de resolver. El procedimiento que se siguió para la optimización del problema de la asignación de ambulancias médicas a un centro asistencial; de un programa matemático lineal y no lineal con programación dinámica, con cierto grado de certidumbre, fue el siguiente: se establecieron las etapas, definiendo las alternativas en cada etapa y los estados en cada etapa como cantidades de recursos que se asignan a la etapa actual y a las etapas subsecuentes; esto dio lugar a la realización de un algoritmo matemático para la obtención de un modelo matemático o función recursiva para cada problema individual. A través del uso del principio de Optimalidad se pudo establecer una recurrencia en avance o en reversa, ya que varian en cuanto a naturaleza y complejidad, dependiendo de la estructura del problema. El resultado que se obtuvo fue la asignación de 1,2,y2, ambulancias médicas a cada centro asistencial y un punto óptimo con un valor óptimo para el problema lineal. Asimismo, en la optimización de programas no lineales con programación dinámica se hizo uso de un recurso muy útil en las matemáticas, extremo condicionado, necesario para la obtención de un punto óptimo.

Palabras clave: Programación dinámica deterministica, función recursiva en avance o reversa.

\section{ABSTRACT}

The deterministic dynamic programming is an optimization method very useful for decomposing large complex mathematical programs in stages, where each stage includes a subproblem of a single variable that is easier to solve individually. The procedure followed for the optimization problem of allocating medical ambulance to a hospital; of linear and nonlinear mathematical program with dynamic programming, with some degree of certainty, was the following: the stages were established, defining the alternatives at each stage and the states in each stage as amounts of resources allocated to the present stage and subsequent stages; this resulted in the completion of a mathematical algorithm to obtain a mathematical model or recursive function for each individual problem. By using the principle of optimality allowed to establish a recurrence forward or reverse since they vary in nature and complexity, depending on the structure of the problem. The result obtained was the allocation of 1,2, and 2, medical ambulances to each health center and an optimal point with an optimal value for the linear problem. Also, in the optimization of nonlinear dynamic programming programs a very useful resource in mathematics, conditional end, was used necessary for obtaining an optimum point.

Keywords: Dynamic programming deterministic, recursive function in forward or reverse

\section{INTRODUCCIÓN}

La primera gran disciplina que surgió a partir del abordaje matemático de los problemas específicos de la guerra fue, seguramente, la Investigación Operativa. El término Operations Research fue utilizado por primera vez en Inglaterra, en 1941. En los años posteriores a la Guerra se abrieron nuevos temas de investigación y se plantearon nuevos problemas que fueron abordados desde una perspectiva matemática. Entre estos nuevos temas se encontraba la teoría de los procesos de decisión en múltiples pasos, que
Richard Bellman abordó alrededor de 1952, y para los cuales fue pensada originalmente la Programación Dinámica. Después de desarrollar el método en el área específica de los problemas de decisión discretos, Bellman y sus colaboradores se dedicaron a la ardua tarea de formular diferentes problemas en los términos de la Programación Dinámica. Como resultado de esta labor, encontraron que las ideas centrales del método, en particular, el Principio de Optimalidad, podían ser aplicadas satisfactoriamente en muchos de los problemas abordados. Descubrieron también las limitaciones de esta técnica y hallaron modos de sobreponerse a

Maestro en Ciencia con mención en Matemáticas. Docente de la Facultad de Ciencias de la Universidad Nacional Jorge Basadre Gronhmann. Tacna-Perú.

${ }^{2}$ Licenciado en Matemáticas. Docente de la Facultad de Ciencias de la Universidad Nacional Jorge Basadre Gronhmann. Tacna - Perú.

${ }^{3}$ Licenciado en Física. Docente de la Facultad de Ciencias de la Universidad Nacional Jorge Basadre Gronhmann. Tacna - Perú.

“ Licenciado en Estadística. Docente de la Facultad de Ciencias de la Universidad Nacional Jorge Basadre Gronhmann. Tacna-Perú. 
Chávez, J. et al. Optimización de programas matemáticos con programación dinámica.

ellas, para algunos problemas puntuales. (Maurete \& Ojea, 2006).

La programación dinámica se basa en el principio de optimalidad, el cual establece que una política óptima está formada por subpolíticas. Pueden definirse como una técnica matemática que da solución a una serie de decisiones secuenciales, cada una de las cuales afecta a las soluciones futuras. Esto es importante, ya que raras veces se encuentra una solución de operaciones en las que las implicaciones de una decisión no se extienden hacia el futuro. Los ejecutivos afrontan soluciones que requieren la toma de una serie de decisiones, en la que el resultado de cada una depende de los resultados de una o unas decisiones previas de la serie. (Thierauf, 1982).

La programación dinámica encuentra la solución óptima de un problema con $|\mathrm{n}|$ variables descomponiéndolo en $|\mathrm{n}|$ etapas, siendo cada etapa un subproblema de una sola variable. Sin embargo, como la naturaleza de la etapa difiere de acuerdo con el problema de optimización, la programación dinámica determinística proporciona los detalles para optimizar cada etapa. La programación dinámica es un método que permite determinar de manera eficiente las decisiones que optimizan el comportamiento de un sistema que evoluciona a lo largo de una serie de etapas (Taha, 1998).

El objetivo fue optimizar programas matemáticos con programación dinámica, con un cierto grado de certidumbre, es decir, maximizar programas matemáticos lineales y no lineales con programación dinámica. Examinar el gran potencial de la programación dinámica determinística debido al proceso de toma de decisiones en varias etapas y presentar la estructura básica para la formulación de un programa lineal o no lineal como un modelo matemático de programación dinámica determinística.

La programación dinámica es un método matemático obtenido mediante un algoritmo matemático de los problemas de optimización. La idea básica del método es descomponer el problema en subproblemas (más pequeños) los cuales son algorítmicamente más manejables. El programa matemático lineal o no lineal es posible optimizarlo definiendo las etapas, las alternativas y los estados en cada etapa y formulando un modelo matemático o función recursiva hacia adelante o en reversa, obtenida a partir de un algoritmo por el principio de optimalidad hasta obtener soluciones óptimas en cada etapa.

La programación dinámica determinística es un método de optimización utilizado para resolver diversos programas matemáticos. Este método llega a la solución trabajando hacia atrás, partiendo del final del problema. Un problema enorme e inmanejable se convierte en una serie de problemas más pequeños y manejables. La optimización de programas matemáticos lineales y no lineales con programación dinámica determinística es, hoy en día, un recurso imprescindible de la matemática aplicada y, también, una importante herramienta teórica.

\section{MATERIAL Y MÉTODOS}

\section{Materiales}

Se consideró como material de estudio programas matemáticos lineales y no lineales que admiten la siguiente formulación general con una función objetivo y un conjunto de restricciones, es decir:

$$
\begin{aligned}
& \text { Opt } \mathrm{f}\left(x_{1}, \ldots, x_{n}\right) \\
& \text { s.a }: \\
& \mathrm{h}_{1}\left(x_{1}, \ldots, x_{n}\right)=0 \\
& \vdots \\
& h_{m}\left(x_{1}, \ldots, x_{n}\right)=0 \\
& g_{1}\left(x_{1}, \ldots, x_{n}\right) \leq 0 \\
& \vdots \\
& g_{k}\left(x_{1}, \ldots, x_{n}\right) \leq 0 \\
& \vec{x}=\left(x_{1}, \ldots, x_{n}\right) \in S \subset R^{n}
\end{aligned}
$$

$$
\text { donde } \boldsymbol{f}, h_{i}, g_{j}: R^{n} \rightarrow R, i=1, m, j=1, k(m<n)
$$

Los elementos de un programa matemático son los siguientes:

$\left(\mathrm{x}_{1}, \ldots, \mathrm{x}_{\mathrm{n}}\right)$ : Variables de decisión.

$\mathrm{f}\left(\mathrm{x}_{1}, \ldots, \mathrm{x}_{\mathrm{n}}\right)$ : Función objetivo.

Opt: Optimiza la función f que consiste en encontrar su máximo y mínimo.

$\mathrm{h}_{\mathrm{m}}\left(\mathrm{x}_{1}, \ldots, \mathrm{x}_{\mathrm{n}}\right)=0$ : Restricciones de igualdad que han de cumplir las posibles soluciones.

$\mathrm{g}_{k}\left(\mathrm{x}_{1}, \ldots, \mathrm{x}_{n}\right) \leq 0$ : Restricciones de desigualdad que han de cumplir las posibles soluciones.

$\left(\mathrm{x}_{1}, \ldots, \mathrm{x}_{\mathrm{r}}\right) \in \mathrm{S}$ : Restricciones conjuntistas.

Los elementos básicos de la programación dinámica, con cierto grado de certeza, son los siguientes:

\section{Definición de las etapas}

Establece las etapas del proceso. En ocasiones, cada etapa es el resultado de una evolución natural del modelo a lo largo del tiempo y en otras ocasiones estas etapas tienen más que ver con el razonamiento empleado para resolver el problema que con una verdadera evolución temporal del sistema.

\section{Definición de los estados en cada etapa}

Variable de la información necesaria para conocer la evolución del sistema a partir de ese momento. Dicha evolución dependerá de los valores de estado y de los valores de la variable de decisión.

\section{Definición de las variables de decisión en cada etapa}

Variable de decisión son oportunidades que pueden tomarse en un determinado estado. Dependiendo del modelo matemático, el rango de valores que pueden tomar la variable de decisión puede depender del estado en que se encuentre el sistema.

\section{Definición de la función recursiva.}

Función que no sólo busca optimizar, sino resolver el modelo de programación dinámica para que sea operativo el modelo de recurrencia.

\section{Principio de Optimalidad}

El enunciado es el siguiente: "Una política óptima tiene la propiedad de que, sean cuales sea el estado inicial, las 
Chávez, J. et al. Optimización de programas matemáticos con programación dinámica.

decisiones restantes deben constituir una solución óptima con respecto al estado resultante de la primera decisión" (Bellman, 1957).

Lo que significa que toda solución al problema total (política óptima) está compuesta de subproblemas (subpolítica) que es también solución óptima de dicho subproblema.

\section{Métodos}

Se utilizó el método recursivo en avance o en reversa, Se caracteriza porque el estado en la siguiente etapa queda completamente determinado por el estado y la política de decisión de la etapa actual. El método recursivo en avance es utilizado para desarrollar los cálculos de la etapa 1, hallando la solución óptima para esa etapa, hasta encontrar la solución óptima de la etapa $n$, ya que la solución óptima de un subproblema se usa como dato para el siguiente subproblema. El método recursivo en reversa comienza en la etapa n, hasta encontrar la solución óptima en la etapa 1. En cada etapa se define la variable de decisión, el valor de retorno de cada variable de decisión, el estado de entrada y de salida y la solución óptima en el estado de dicha etapa. Asimismo, en cada etapa, la función recursiva en avance o en reversa es un modelo matemático obtenido a partir de un algoritmo matemático.

El modelo matemático de la función o ecuación recursiva en reversa para el problema de las distribuciones de ambulancias es:

$$
f_{n}^{*}\left(x_{n}\right)=\min _{1 \leq d_{n} \leq 3}\left\{R_{n}\left(d_{n}\right)+f_{n+1}^{*}\left(x_{n+1}\right)\right\}
$$

El modelo matemático de la ecuación recursiva en avance para el problema lineal es:

$$
f_{i}^{*}\left(s_{i}, t_{i}, u_{i}\right)=\max _{d_{i}}\left\{R_{i}\left(d_{i}\right)+f_{i-1}^{*}\left(x_{i-1}\right) \equiv 0\right\}
$$

Modelo matemático de la ecuación recursiva en avance para el problema no lineal es:

$$
f_{i}^{*}\left(s_{i}, t_{i}\right)={ }_{d_{i}}^{\max }\left\{R_{i}\left(d_{i}\right)+f_{i-1}^{*}\left(s_{i-1}, t_{i-1}\right), f_{i-1}^{*}\left(s_{i-1}, t_{i-1}\right) \equiv 0\right\}
$$

\section{RESULTADOS}

\section{Distribución de ambulancias médicas con programa- ción dinámica deterministica}

Un proyecto del Ministerio de Salud requirió entregar 5 ambulancias a 3 centros asistenciales en el interior del país. En la tabla 1 se determinó la respuesta en tiempo promedio de llegada a un punto de socorro, al contar con 1, 2 y 3 ambulancias. Se condicionó el reparto de las ambulancias para que al menos un vehículo corresponda a cada centro asistencial.

Tabla 1. Tiempo promedio de las ambulancias de llegar a un punto de socorro.

\begin{tabular}{cccc}
\hline \multirow{2}{*}{ Ambulancias Médicas } & \multicolumn{3}{c}{ Centros Asistenciales } \\
& $\mathbf{1}$ & $\mathbf{2}$ & $\mathbf{3}$ \\
\hline 1 & 8 & 10 & 12 \\
2 & 6 & 5 & 6 \\
3 & 3 & 4 & 5 \\
\hline
\end{tabular}

Este problema tuvo 3 variables decisionales y como objetivo, una función que minimiza el tiempo total de respuesta. En la tabla 2 se muestra el problema de las ambulancias como un modelo de programación dinámica determinística para una función en reversa y el resultado fue que a cada centro asistencial le asignaron 1,2,2 ambulancias médicas.

Tabla 2. Asignación de ambulancias medicas como un modelo de programación determinística.

\begin{tabular}{ccccccc}
\hline & $\mathrm{d}_{1}=\mathbf{1}$ & & $\mathrm{d}_{2}=\mathbf{2}$ & \multicolumn{3}{c}{$\mathrm{d}_{3}=\mathbf{2}$} \\
\hline $\mathrm{x}_{1}=5$ & Etapa 1 & $\mathrm{x}_{2}=4$ & Etapa 2 & $\mathrm{x}_{3}=2$ & Etapa 3 & $\mathrm{x}_{4}=0$ \\
& $\mathrm{R}_{1}\left(\mathrm{~d}_{1}\right)=8$ & & $\mathrm{R}_{2}\left(\mathrm{~d}_{2}\right)=5$ & & $\mathrm{R}_{3}\left(\mathrm{~d}_{3}\right)=6$ & \\
\hline
\end{tabular}

Resolución de un programa lineal usando programación dinámica determinística

Usando la programación dinámica determinística se resolvió el programa matemático lineal:

$$
\left.\begin{array}{r}
\max z=2 x+3 y \\
\text { s.a: }-3 x+2 y \leq 6 \\
2 x+y \leq 10 \\
2 x-y \leq 6 \\
x \geq 0, y \geq 0
\end{array}\right\}
$$

En la tabla 3 se muestra el programa lineal como un modelo de programación dinámica determinística para una función recursiva en avance.

Tabla 3. Estructura de un modelo lineal con programación dinámica determinística.

\begin{tabular}{ccccc}
\hline & $\mathrm{d}_{2}=\mathbf{x}_{2}$ & \multicolumn{3}{c}{$\mathrm{d}_{1}=\mathbf{x}_{1}$} \\
\hline $\mathrm{s}_{2}=6$ & & $\mathrm{~s}_{1}=\mathrm{s}_{2}-2 \mathrm{~d}_{2}$ & & $\mathrm{~s}_{0}=\mathrm{s}_{1}+3 \mathrm{~d}_{t}$ \\
$\mathrm{t}_{2}=10$ & Etapa 2 & $\mathrm{t}_{1}=\mathrm{t}_{2}-\mathrm{d}_{2}$ & Etapa 1 & $\mathrm{t}_{1}=\mathrm{t}_{1}-2 \mathrm{~d}_{1}$ \\
$\mathrm{u}_{2}=6$ & & $\mathrm{u}_{1}=\mathrm{u}_{2}+\mathrm{d}_{2}$ & & $\mathrm{u}_{0}=\mathrm{u}_{1}-2 \mathrm{~d}_{1}$ \\
& & & $\mathrm{R}_{1}\left(\mathrm{~d}_{)}\right)=2 \mathrm{x}_{1}$ & \\
\hline
\end{tabular}

El resultado que se obtuvo fue de un punto óptimo de $(2 ; 6)$, con un valor de óptimo de 22 .

\section{Resolución de un programa no lineal usando progra- mación dinámica determinística}

Mediante la programación dinámica se resolvió el programa matemático no lineal.

$$
\begin{array}{r}
\max z=7 x^{2}+6 x+5 y^{2} \\
\text { s.a: } x+2 y \leq 10 \\
x-3 y \leq 9 \\
x, y \geq 0
\end{array}
$$

En la tabla 4 se muestra el programa no lineal como un modelo de programación dinámica determinística para una recursiva en avance:

Tabla 4. Estructura de un modelo no lineal con programación dinámica determinística.

\begin{tabular}{ccccc}
\hline \multicolumn{2}{c}{$\mathbf{d}_{2}=\mathbf{x}_{2}$} & \multicolumn{3}{c}{$\mathbf{d}_{1}=\mathbf{x}_{1}$} \\
\\
\hline $\mathrm{s}_{2}=10$ & Etapa 2 & $\mathrm{s}_{1}=\mathrm{s}_{2}-2 \mathrm{~d}_{2}$ & Etapa 1 & $\mathrm{s}_{\mathrm{n}}=\mathrm{s}_{1}-\mathrm{d}_{1}$ \\
$\mathrm{t}_{2}=9$ & & $\mathrm{t}_{1}=\mathrm{t}_{2}+3 \mathrm{~d}_{2}$ & & $\mathrm{t}_{0}=\mathrm{t}_{1}-\mathrm{d}_{1}$ \\
& $\mathrm{R}_{2}\left(\mathrm{~d}_{2}\right)=5 \mathrm{~d}_{2}^{2}$ & & $\mathrm{R}_{1}\left(\mathrm{~d}_{1}\right)=7 \mathrm{~d}_{1}^{2}+6 \mathrm{~d}_{1}$ & \\
& & & &
\end{tabular}




\section{Ciencia \& Desarrollo}

Chávez, J. et al. Optimización de programas matemáticos con programación dinámica.

El resultado que se obtuvo fue de un punto óptimo donde: de $(9,6 ; 0,2)$, con un valor óptimo de 702,92.

\section{DISCUSIÓN}

Distribución de ambulancias médicas con programación dinámica determinística

Un proyecto del Ministerio de Salud requiere entregar 5 ambulancias a 3 centros asistenciales en el interior del país. Se ha determinado que la respuesta en el tiempo promedio de llegada a un punto de socorro, según la tabla 5 , al contar con 1, 2 y 3 ambulancias. Se ha condicionado el reparto de las ambulancias para que al menos un vehículo corresponda a cada centro asistencial. Este problema tiene 3 variables decisionales y como objetivo una función que minimiza el tiempo total de respuesta ¿cuántas ambulancias le toca a cada centro asistencial?

Tabla 5. Tiempo promedio de llegar a un punto de socorro.

\begin{tabular}{cccc}
\hline $\begin{array}{c}\text { Ambulancias } \\
\text { Médicas }\end{array}$ & $\mathbf{1}$ & Centros Asistenciales \\
\hline 1 & 8 & $\mathbf{2}$ & $\mathbf{3}$ \\
\hline 2 & 6 & 10 & 12 \\
3 & 3 & 5 & 6 \\
\hline
\end{tabular}

Para resolver el problema usando programación dinámica, con cierto grado de certidumbre, se debe tener en cuenta los siguientes pasos finitos consecutivos:

Primer paso. Se diagrama los tiempos promedios para una ecuación recursiva en reversa la cual se muestra en la figura 1.

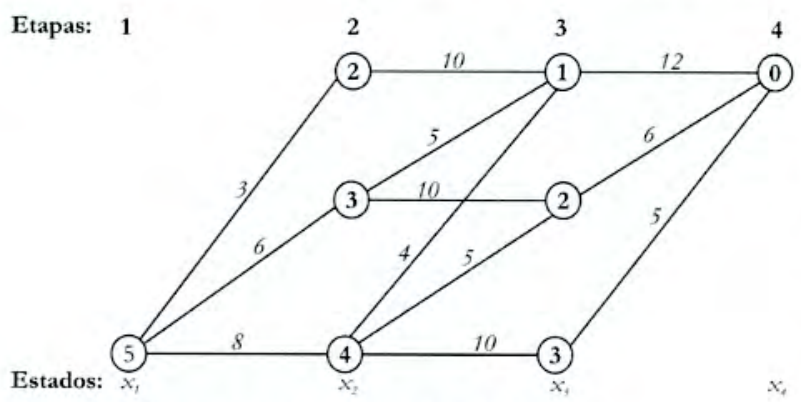

Figura 1. Tiempos promedios en reversa.

Segundo paso. Describimos el programa matemático con el objetivo de elegir $\mathrm{d}_{1}, \mathrm{~d}_{2}, \mathrm{~d}_{3}$ para:

$$
\left.\begin{array}{l}
\min \sum_{i=1}^{3} R_{i}\left(d_{i}\right)=R_{1}\left(d_{1}\right)+R_{2}\left(d_{2}\right)+R_{3}\left(d_{3}\right) \\
\text { s.a: } \sum_{i=1}^{3} d_{i}=d_{1}+d_{2}+d_{3} \\
d_{i} \text { entero positivo }
\end{array}\right\}
$$

Tercer paso. El modelo matemático de la función o ecuación recursiva en reversa queda expresada como:

$$
f_{n}^{*}\left(x_{n}\right)=\min _{1 \leq d_{n} \leq 3}\left\{R_{n}\left(d_{n}\right)+f_{n+1}^{*}\left(x_{n+1}\right)\right\}
$$

$$
\begin{aligned}
& f_{n}\left(x_{n}, d_{n}\right)=R_{n}\left(d_{n}\right)+f_{n+1}^{*}\left(x_{n+1}\right) \\
& x_{n+1}=x_{n}-d_{n}
\end{aligned}
$$

Cuarto paso. Cálculo de la ecuación recursiva en cada una de las etapas:

$$
\begin{aligned}
& n=3 \\
& f_{3}^{*}\left(x_{3}\right)=\min _{1 \leq d_{3} \leq 3}\left\{R_{3}\left(d_{3}\right)+f_{4}^{*}\left(x_{4}\right)\right\}
\end{aligned}
$$

en donde :

$$
\begin{aligned}
f_{3}\left(x_{3}, d_{3}\right) & =R_{3}\left(d_{3}\right)+f_{4}^{*}\left(x_{4}\right) \\
x_{4} & =x_{3}-d_{3} \\
x_{3} & =d_{3}
\end{aligned}
$$

Tabla 6. Cálculos para $\mathrm{f}_{3}{ }^{*}\left(\mathrm{x}_{3}\right)$.

\begin{tabular}{cccccc}
\hline $\mathbf{d}_{3}$ & & $\mathbf{f}_{3}\left(\mathbf{x}_{3}, \mathbf{d}_{3}\right)=\mathbf{R}_{3}\left(\mathbf{d}_{3}\right)$ & \multicolumn{2}{c}{ Solución Óptima } \\
$\mathbf{x}_{3}$ & $\mathbf{1}$ & $\mathbf{2}$ & $\mathbf{3}$ & $\mathbf{f}_{3}^{*}\left(\mathbf{x}_{3}\right)$ & $\mathbf{d}_{3}^{*}$ \\
\hline 1 & 12 & - & - & 1 & 1 \\
2 & - & 6 & - & 6 & 2 \\
3 & - & - & 5 & 5 & 3 \\
\hline
\end{tabular}

$$
n=2
$$

$f_{2}^{*}\left(x_{2}\right)=\min _{1 \leq d_{2} \leq 3}\left\{R_{2}\left(d_{2}\right)+f_{3}^{*}\left(x_{3}\right)\right\}$

en donde:

$\begin{aligned} f_{2}\left(x_{2}, d_{2}\right) & =R_{2}\left(d_{2}\right)+f_{3}^{*}\left(x_{3}\right) \\ x_{3} & =x_{2}-d_{2}\end{aligned}$

$x_{2}=2$

$d_{2}=1: f_{2}(2,1)=R_{2}(1)+f_{3}^{*}(1)=10+12=22$

$x_{2}=3$

$d_{2}=1: f_{2}(3,1)=R_{2}(1)+f_{3}^{*}(2)=10+6=16$

$d_{2}=2: f_{2}(3,2)=R_{2}(2)+f_{3}^{*}(1)=5+12=17$

$x_{2}=4$

$d_{2}=1: f_{2}(4,1)=R_{2}(1)+f_{3}^{*}(3)=10+5=15$

$d_{2}=2: f_{2}(4,2)=R_{2}(2)+f_{3}^{*}(2)=5+6=11$

$d_{2}=3: f_{2}(4,3)=R_{2}(3)+f_{3}^{*}(1)=4+12=16$

Tabla 7. Cálculos para $\mathrm{f}_{2}^{\prime}\left(\mathrm{x}_{2}\right)$.

\begin{tabular}{cccccc}
\hline & $\mathbf{d}_{2}$ & \multicolumn{2}{c}{$\mathbf{f}_{2}\left(\mathbf{x}_{2}, \mathbf{d}_{2}\right)=\mathbf{R}_{2}\left(\mathbf{d}_{2}\right)+\mathbf{d}_{3}^{*}\left(\mathbf{x}_{3}\right)$} & \multicolumn{2}{c}{ Solución Óptima } \\
$\mathbf{x}_{2}$ & $\mathbf{1}$ & $\mathbf{2}$ & $\mathbf{3}$ & $\mathbf{f}_{2}^{*}\left(\mathbf{x}_{2}\right)$ & $\mathbf{d}_{2}^{*}$ \\
\hline 2 & 22 & - & - & 22 & 1 \\
3 & 16 & 17 & - & 16 & 1 \\
4 & 15 & 11 & 16 & 11 & 2 \\
\hline
\end{tabular}

$n=1$

$f_{1}^{*}\left(x_{1}\right)=\min _{1 \leq d_{1} \leq 3}\left\{R_{1}\left(d_{1}\right)+f_{2}^{*}\left(x_{2}\right)\right\}$

en donde :

$f_{1}\left(x_{1}, d_{1}\right)=R_{1}\left(d_{1}\right)+f_{2}^{*}\left(x_{2}\right)$

$x_{2}=x_{1}-d_{1}$ 


$$
\begin{aligned}
& x_{1}=5 \\
& d_{1}=1: f_{1}(5,1)=R_{1}(1)+f_{2}^{*}(4)=8+11=19 \\
& d_{1}=2: f_{1}(5,2)=R_{1}(2)+f_{2}^{*}(3)=6+16=22 \\
& d_{1}=3: f_{1}(5,3)=R_{1}(3)+f_{2}^{*}(2)=3+22=25
\end{aligned}
$$

Tabla 8. Cálculos para $\mathrm{f}_{1}^{*}\left(\mathrm{x}_{1}\right)$.

\begin{tabular}{ccccccc}
\hline & $\mathbf{d}_{1}$ & \multicolumn{2}{c}{$\mathbf{f}_{1}\left(\mathbf{x}_{1}, \mathbf{d}_{1}\right)=\mathbf{R}_{1}\left(\mathrm{~d}_{1}\right)+\mathbf{f}_{2}^{*}\left(\mathbf{x}_{2}\right)$} & \multicolumn{2}{c}{ Solución Óptima } \\
$\mathbf{x}_{1}$ & $\mathbf{1}$ & $\mathbf{2}$ & $\mathbf{3}$ & $\mathbf{f}_{1}^{*}\left(\mathbf{x}_{1}\right)$ & $\mathbf{d}_{1}^{*}$ \\
\hline 5 & 19 & 22 & 25 & 19 & 1 \\
\hline
\end{tabular}

Quinto paso. Asignación de ambulancias médicas:

$$
\left.\left.\begin{array}{rl}
x_{2} & =x_{1}-d_{1} \\
x_{1}=5 & \left.\begin{array}{r}
x_{2} \\
d_{1}=1
\end{array}\right\} \begin{array}{l}
x_{3}=x_{2}-d_{2} \\
x_{2}
\end{array} \\
d_{2}=4 & x_{3}=4-2 \\
x_{3}=2 \\
d_{3}=2
\end{array}\right\} \begin{array}{l}
x_{4}=x_{3}-d_{3} \\
x_{4}=2-2 \\
x_{4}=0
\end{array}\right\}
$$

Sexto paso. En la tabla 9 se muestra la asignación de ambulancias médicas como un modelo matemático de programación dinámica determinística:

\section{Etapas: 1,2,3.}

Variable de decisión en cada etapa: $\mathrm{d}_{1}, \mathrm{~d}_{2}, \mathrm{~d}_{3}$.

\begin{tabular}{|c|c|c|c|c|c|c|}
\hline & $d_{1}=1$ & & $d_{2}=2$ & & $d_{3}=2$ & \\
\hline$x_{1}=5$ & Etapa 1 & $x_{2}=4$ & Etapa 2 & $x_{3}=2$ & Etapa 3 & $x_{4}=0$ \\
\hline
\end{tabular}

Estado de entrada y de salida en cada etapa: $\mathrm{x}_{1}, \mathrm{x}_{2}, \mathrm{x}_{3}, \mathrm{x}_{4}$.

Valor generado por cada variable de decisión: $\mathrm{R}_{1}, \mathrm{R}_{2}$.

Tabla 9. Asignación de ambulancias médicas como un modelo de programación determinística.

Un punto óptimo es $\left(\mathrm{d}_{1}, \mathrm{~d}_{2}, \mathrm{~d}_{3}\right)=(1 ; 2 ; 2)$ y el valor óptimo del programa matemático es:

$$
\mathrm{R}_{1}\left(\mathrm{~d}_{1}\right)+\mathrm{R}_{2}\left(\mathrm{~d}_{2}\right)+\mathrm{R}_{3}\left(\mathrm{~d}_{3}\right)=8+5+6=19
$$

\section{Optimización de programas lineales con programa-} ción dinámica determinística

Para resolver el programa lineal, usando programación dinámica, con cierto grado de certidumbre, se debe tener en cuenta los pasos finitos consecutivos siguientes:

$$
\left.\begin{array}{r}
\max z=2 x+3 y \\
\text { s.a: }-3 x+2 y \leq 6 \\
2 x+y \leq 10 \\
2 x-y \leq 6 \\
x \geq 0, y \geq 0
\end{array}\right\}
$$

Primer paso. Se establece la estructura básica de un modelo con programación dinámica determinística:

\section{Etapas: 1,2.}

Variable de decisión en cada etapa: $\mathrm{d}_{1}, \mathrm{~d}_{2}$.

Estado de entrada y de salida en cada etapa: $\mathrm{s}_{2}, \mathrm{~s}_{1}, \mathrm{~s}_{0}, \mathrm{t}_{2}, \mathrm{t}_{1}, \mathrm{t}_{1}, \mathrm{u}_{2}, \mathrm{u}_{1}, \mathrm{u}_{0}$ Valor generado por cada variable de decision: $\mathrm{R}_{2}, \mathrm{R}_{1}$
Segundo paso. Se plantea el programa lineal como un modelo matemático de programación dinámica determinística:

Tabla 10. Estructura de un programa lineal con programación dinámica determinística.

\begin{tabular}{ccrcc}
\hline & $\mathrm{d}_{2}=\mathrm{x}_{2}$ & \multicolumn{3}{c}{$\mathrm{d}_{1}=\mathrm{x}_{1}$} \\
\hline $\mathrm{s}_{2}=6$ & Etapa 2 & $\mathrm{s}_{1}=\mathrm{s}_{2}-2 \mathrm{~d}_{2}$ & Etapa 1 & $\mathrm{s}_{0}=\mathrm{s}_{1}+3 \mathrm{~d}_{1}$ \\
$\mathrm{t}_{2}=10$ & & $\mathrm{t}_{1}=\mathrm{t}_{2}-\mathrm{d}_{2}$ & & $\mathrm{t}_{0}=\mathrm{t}_{1}-2 \mathrm{~d}_{1}$ \\
$\mathrm{u}_{2}=6$ & & $\mathrm{u}_{1}=\mathrm{u}_{2}+\mathrm{d}_{2}$ & & $\mathrm{u}_{1}=\mathrm{u}_{1}-2 \mathrm{~d}_{1}$ \\
& $\mathrm{R}_{2}\left(\mathrm{~d}_{2}\right)=3 \mathrm{x}_{2}$ & & $\mathrm{R}_{1}\left(\mathrm{~d}_{1}\right)=2 \mathrm{x}_{1}$ & \\
\end{tabular}

Tercer paso. Se determina el modelo matemático de la función o ecuación recursiva en avance:

$$
\begin{aligned}
f_{i}^{*}\left(s_{i}, t_{i}, u_{i}\right) & =\max _{d i}\left\{R_{i}\left(d_{i}\right)+f_{i-1}^{*}\left(x_{i-1}\right) ; f_{i-1}^{*}\left(x_{i-1}\right) \equiv 0\right\} \\
& =\max _{d i}\left\{R_{i}\left(d_{i}\right)+f_{i-1}^{*}\left(s_{i}, t_{i}, u_{i}\right) ; f_{i-1}^{*}\left(s_{i}, t_{i}, u_{i}\right) \equiv 0\right\}
\end{aligned}
$$

donde las ecuaciones de los estados o restricciones son:

$$
\begin{aligned}
\mathrm{s}_{\mathrm{i}-1} & =\mathrm{s}_{\mathrm{i}}-\mathrm{a}_{1 \mathrm{i}} \mathrm{d}_{\mathrm{i}} \\
\mathrm{t}_{\mathrm{i}-1} & =\mathrm{t}_{\mathrm{i}}-\mathrm{a}_{2} \mathrm{~d}_{\mathrm{i}} \\
\mathrm{u}_{\mathrm{i}-1} & =\mathrm{u}_{\mathrm{i}}-\mathrm{a}_{31} \mathrm{~d}_{\mathrm{i}}
\end{aligned}
$$

Cuarto paso. Se calcula la ecuación recursiva en cada una de las etapas:

$$
\begin{aligned}
i=1 & \begin{aligned}
f_{1}^{*}\left(s_{1}, t_{1}, u_{1}\right)= & \max _{d_{1}}\left\{R_{1}\left(d_{1}\right)+f_{0}^{*}\left(s_{0}, t_{0}, u_{0}\right)\right\} \\
& ={ }^{\max }\left\{2 d_{1}\right\} \ldots \text { (1) }
\end{aligned}
\end{aligned}
$$

Se sabe que

$s_{0} \geq 0 \Rightarrow s_{1}+3 d_{1} \geq 0 \Rightarrow-s_{1} / 3 \leq d_{1} \leq 0$

$t_{0} \geq 0 \Rightarrow t_{1}-2 d_{1} \geq 0 \Rightarrow 0 \leq d_{1} \leq t_{1} / 2$

$u_{0} \geq 0 \Rightarrow u_{1}-2 d_{1} \geq 0 \Rightarrow 0 \leq d_{1} \leq u_{1} / 2$

Ocurre un máximo en la ecuación (1)

$$
\begin{aligned}
& \operatorname{si} d_{1}=\min \left(\frac{t_{1}}{2}, \frac{u_{1}}{2}\right) \text {, entonces } \\
& f_{1}^{*}\left(\mathrm{~s}_{1}, \mathrm{t}_{1}, \mathrm{u}_{1}\right)=2 * \min \left\{\frac{t_{1}}{2}, \frac{u_{1}}{2}\right\}
\end{aligned}
$$

$$
\begin{aligned}
i=2 & \\
f_{2}^{*}\left(s_{2}, t_{2}, u_{2}\right) & ={ }_{d_{2}}^{\max }\left\{R_{2}\left(d_{2}\right)+f_{1}^{*}\left(s_{1}, t_{1}, u_{1}\right)\right\} \\
& ={ }^{\max }\left\{3 d_{2}+2 * \min \left\{t_{1} / 2, u_{1} / 2\right\}\right\} \\
& ={ }^{\max }\left\{3 d_{2}+2 * \min \left\{\left(t_{2}-d_{2}\right) / 2,\left(u_{2}+d_{2}\right) / 2\right\}\right\} \\
& ={ }^{\max }\left\{3 d_{2}+2 * \min \left\{\left(10-d_{2}\right) / 2,\left(6+d_{2}\right) / 2\right\}\right\}
\end{aligned}
$$

Quinto paso. Se calcula el valor óptimo

Si $0 \leq d_{2} \leq 10$, entonces $d_{2}=6$

Se sabe que $t_{1}=10-6=4 ; u_{1}=6+6=12$

Luego ocurre un máximosi $d_{1}=\min \left(\frac{4}{2}, \frac{12}{2}\right)=2$ 
Chávez, J. et al. Optimización de programas matemáticos con programación dinámica.

Por lo tanto, un punto óptimo es $\left(\mathrm{d}_{1}, \mathrm{~d}_{2}\right)=(2 ; 6)$. Finalmente el valor óptimo del programa matemático es:

$$
z=2(2)+3(6)=22
$$

\section{Optimización de Programas no lineales con programa-} ción dinámica determinística

Para resolver el siguiente programa no lineal usando programación dinámica, con cierto grado de certidumbre, se debe tener en cuenta los siguientes pasos finitos consecutivos:

$$
\left.\begin{array}{r}
\max z=7 x^{2}+6 x+5 y^{2} \\
s \cdot a: x+2 y \leq 10 \\
x-3 y \leq 9 \\
x, y \geq 0
\end{array}\right\}
$$

Primer Paso. Se establece el elemento básico del modelo de programación dinámica determinística:

\section{Etapas: 1,2 .}

Variable de decisión en cada etapa: $\mathrm{d}_{1} \mathrm{~d}_{2}$

Estado de entrada y de salida en cada etapa: $\mathrm{s}_{2}, \mathrm{~s}_{1}, \mathrm{~s}_{0}, \mathrm{t}_{2}, \mathrm{t}_{1}, \mathrm{t}_{0}$. Valorgenerado porcada variable de decision: $\mathrm{R}_{2}, \mathrm{R}_{1}$.

Segundo Paso. Se plantea el programa matemático no lineal como un problema de programación dinámica determinística:

Tabla 11. Estructura de un programa no lineal con programación dinámica determinística.

\begin{tabular}{lcccc}
\hline \multicolumn{3}{c}{$\mathrm{d}_{2}=\mathbf{x}_{2}$} & \multicolumn{3}{c}{$\mathrm{d}_{1}=\mathbf{x}_{1}$} \\
\hline $\mathrm{s}_{2}=10$ & Etapa 2 & $\mathrm{s}_{1}=\mathrm{s}_{2}-2 \mathrm{~d}_{2}$ & Etapa 1 & $\mathrm{s}_{0}=\mathrm{s}_{1}-\mathrm{d}_{1}$ \\
$\mathrm{t}_{2}=9$ & & $\mathrm{t}_{1}=\mathrm{t}_{2}+3 \mathrm{~d}_{2}$ & & $\mathrm{t}_{0}=\mathrm{t}_{1}-\mathrm{d}_{1}$ \\
& $\mathrm{R}_{2}\left(\mathrm{~d}_{2}\right)=5 \mathrm{~d}_{2}^{2}$ & & $\mathrm{R}_{1}\left(\mathrm{~d}_{1}\right)=7 \mathrm{~d}_{1}^{2}+6$ & \\
\hline
\end{tabular}

Tercer paso. Se calcula el modelo matemático de la ecuación recursiva en avance:

$f_{1}^{*}\left(s_{i}, t_{1}\right)={ }_{d_{i}}\left\{R_{i}\left(d_{i}\right)+f_{i-1}^{*}\left(s_{i-1}, t_{i-1}\right), f_{i-1}\left(s_{i-1}, t_{i-1}\right) \equiv 0\right\}$

donde las ecuaciones de los estados, restricciones, son:

$$
\begin{aligned}
& \mathrm{s}_{\mathrm{i}-1}=\mathrm{s}_{\mathrm{i}}-\mathrm{a}_{1 \mathrm{i}} \mathrm{d} \\
& \mathrm{t}_{\mathrm{i} 1}=\mathrm{t}_{\mathrm{i}}-\mathrm{a}_{2} \mathrm{~d}_{\mathrm{i}}
\end{aligned}
$$

Cuarto paso. Se calcula de la ecuación recursiva en cada una de las etapas:

$$
\begin{aligned}
& i=1 \\
& f_{1}^{*}\left(s_{1}, t_{1}\right)={ }_{d_{1}}^{\max }\left\{R_{1}\left(d_{1}\right)+f_{0}^{*}\left(s_{0}, t_{0}\right)\right\} \\
& ={ }_{d_{1}}^{m a ́ x}\left\{7 d_{1}^{2}+6 d_{1}+f_{0}^{*}\left(s_{0}, t_{0}\right)\right\} \\
& ={ }_{d_{1}}^{\max }\left\{7 d_{1}^{2}+6 d_{1}\right\}
\end{aligned}
$$

Se sabe que:

$$
\begin{aligned}
& s_{0} \geq 0 \Rightarrow s_{1}-d_{1} \geq 0 \Rightarrow 0 \leq d_{1} \leq s_{1} \\
& t_{0} \geq 0 \Rightarrow t_{1}-d_{1} \geq 0 \Rightarrow 0 \leq d_{1} \leq t_{1}
\end{aligned}
$$

Ocurre un máximo en la ecuación (1) $\operatorname{sid}_{1}=\min \left(\mathrm{s}_{1}, \mathrm{t}_{1}\right)$, entonces:

$$
\begin{aligned}
& f_{1}^{*}\left(s_{1}, t_{1}\right)=7 *\left(\min \left\{s_{1}, t_{1}\right\}\right)^{2}+6^{*} \min \left\{s_{1}, t_{1}\right\} \\
& i=2 \\
& f_{2}^{*}\left(s_{2}, t_{2}\right)={ }_{d_{2}}^{\max }\left\{R_{2}\left(d_{2}\right)+f_{1}^{*}\left(s_{1}, t_{1}\right)\right\} \\
& =\max ^{\prime}\left\{\begin{array}{l}
\left\{\begin{array}{l}
5 d_{2}^{2}+7 *\left\{\min \left\{s_{2}-2 d_{2}, t_{2}+3 d_{2}\right\}\right. \\
+6 * \min \left\{s_{2}-2 d_{2}, t_{2}+3 d_{2}\right\}
\end{array}\right\} \\
d_{2}
\end{array}\right\}
\end{aligned}
$$

Quinto paso. Se reformuló el programa matemático no lineal:

$$
\left.\begin{array}{r}
\max z=7 x^{2}+6 x+5 y^{2} \\
\text { s. } a: x+2 y \leq 10 \\
x-3 y \leq 9 \\
x, y \geq 0
\end{array}\right\}
$$

Para obtener los extremos condicionados sujeta a condiciones de enlace, se procede primero construyendo la función de Lagrange:

$$
\begin{aligned}
& L\left(x, y, \lambda_{1}, \lambda_{2}\right)=7 x^{2}+6 x+5 y^{2}+\lambda_{1}(x+2 y-10)+\lambda_{2}(x-3 y-9) \\
& \frac{\partial L}{\partial x}=14 x+6+\lambda_{1}+\lambda_{2}=0 \ldots(2) \\
& \frac{\partial L}{\partial y}=10 y+2 \lambda_{1}-3 \lambda_{2}=0 \quad \ldots(3) \\
& \frac{\partial L}{\partial \lambda_{1}}=x+2 y-10=0 \\
& \frac{\partial L}{\partial \lambda_{2}}=x-3 y-9=0
\end{aligned}
$$

Resolviendo (4) y (5) se tiene $d_{2}=0,2$. Se sabe que:

$\mathrm{s}_{1}=10-2 \mathrm{~d}_{2}=10-2(0,2)=9,6$ y $\mathrm{t}_{1}=9+3 \mathrm{~d}_{2}=9+3(0,2)=9,6$

Luego ocurre un máximo si $\mathrm{d}_{1}=\min \left\{\mathrm{s}_{1}, \mathrm{t}_{1}\right\}=9,6$. Por lo tanto un punto óptimo es $\left(\mathrm{d}_{1}, \mathrm{~d}_{2}\right)=(\mathrm{x} ; \mathrm{y})=(9,6 ; 0,2)$. Finalmente el valor óptimo del programa no lineal es:

$z=7(9,6)^{2}+6(9,6)+5(0,2)^{2}=702,92$

\section{CONCLUSIONES}

La programación dinámica es un método de optimización que descompone los programas matemáticos de $n$ variable en n etapas, en que cada etapa incluye un subproblema de una sola variable.

La optimización de programas matemáticos con programación dinámica determinística requiere de un método matemático o función recursiva en avance obtenida de un algoritmo matemático apropiado para cada problema individual.

En la optimización de programas matemáticos no lineales con programación dinámica, con cierto grado de certeza, la aplicación de los multiplicadores de Lagrange 
Chávez, J. et al. Optimización de programas matemáticos con programación dinámica.

linealizan el programa no lineal para encontrar valores ópti- Bronson R. (1996). Investigación de operaciones. México: mos. Schaum-McGraw-Hill.

\section{REFERENCIAS BIBLIOGRÁFICAS.}

Ackoff, R. (1987). Fundamentos de Investigación de operaciones. México: Limusa.

Bellman, R. (1957). Dynamic Programming. Princeton: Princeton University Press
Maurette, M., Ojea, I. (2006). Programación Dinámica. Monografía.

Sasieni, M. (1982). Investigación de operaciones. México: Limusa. Taha, A. (1998). Investigación de operaciones. Una introducción. México: Prentice Hall. $6^{\circ}$ Edición.

Thierauf, R. (1986). Introducción a la investigación de operaciones. México: Limusa.

\section{Correspondencia:}

Jhony Alfonso Chávez Delgado: jhonychavez@yahoo.es

Fecha de Recepción: 10/10/2014

Luis César Mendez Ávalos: luismendezavalos@hotmail.com

Fecha de Aceptación: 01/06/2015

Eduardo Rodríguez Delgado: eduardo.rodriguez.delgado@gmail.com

Luis Asunción López Puycán: lulopuy@hotmail.com 